\title{
Percepción de los estudiantes de Comunicación Social sobre la dicotomía ciencias/letras como marco de reflexión para incentivar la difusión de las ciencias
}

\author{
María Dolores Meneses-Fernández*, Silvia Granja-González**, Juana Dolores Santana-Hernández* \\ * Facultad de Ciencias Sociales y de la Comunicación, Universidad de La Laguna \\ e-mail: dmeneses@ull.edu.es | ORCID iD: https://orcid.org/0000-0001-8516-4298 \\ e-mail: jsanther@ull.edu.es | ORCID iD: https://orcid.org/0000-0002-2220-8107 \\ ** Escola de Doctorat, Universitat de València \\ e-mail: silgran@alumni.uv.es | ORCID iD: https://orcid.org/0000-0001-5789-3362
}

Recibido: 01-09-20; 2a versión: 13-11-20; Aceptado: 23-12-20; Publicado: 04-02-22

Cómo citar este artículo/Citation: Meneses-Fernández, M. D.; Granja-González, S.; Santana-Hernández, J. D. (2022). Percepción de los estudiantes de Comunicación Social sobre la dicotomía ciencias/letras como marco de reflexión para incentivar la difusión de las ciencias. Revista Española de Documentación Científica, 45 (1), e314. https://doi.org/10.3989/redc.2022.4.1824

Resumen: El periodismo científico comparte hoy su función con otros emisores de mensajes. Se suma que es una opción profesional minoritaria. Para averiguar el origen de esta situación partimos de que las universidades españolas forman en una estructura académica dicotómica ciencias/letras, en la que los estudiantes de una rama de conocimiento reciben escasa formación sobre los conocimientos de las otras. Analizamos si esta organización académica influye en la percepción que los estudiantes de Comunicación Social tienen de las ciencias experimentales, de la naturaleza, de la salud, de las humanidades y sociales. Para ello encuestamos a estudiantes de Periodismo y de Comunicación Audiovisual utilizando un cuestionario impreso estructurado en preguntas abiertas, dicotómicas y en escala. Luego aplicamos estadística descriptiva a los datos obtenidos, lo que permitió comprobar que los futuros periodistas no solo ingresan en la carrera considerándose de letras, sino que avanzan en ella sin modificar esa autopercepción. Una conclusión es que urge replantear la formación imperante para abrirla a una concepción pedagógica inter y transdisciplinar integradora que mejore la presencia de las ciencias en la agenda informativa.

Palabras Clave: comunicación de las ciencias; periodismo científico; periodismo especializado; formación periodística; formación universitaria integrada.

\section{Social Communication students' perception of the sciences/letters dichotomy as a reflecting framework to promote the dissemination of sciences}

Abstract: Nowadays, science journalism shares its role with other message transmitters. Moreover, it is a minority professional option. To find out the origin of this situation, we start from the proposal that Spanish universities train in a dichotomous academic structure, Science/Letters. In this academic structure, students in one branch of knowledge receive little training on the knowledge of the others. We analyze whether this academic organization influences the perception that Social Communication students have of the experimental sciences, nature, health, humanities and social sciences. To verify it, we surveyed journalism and audiovisual communication students. We used a printed questionnaire structured in open, dichotomous and scale questions. Then we applied descriptive statistics to the obtained data, which allowed us to verify that future journalists not only entered the undergraduate studies considering themselves Letters students, but they went through it without modifying that self-perception. One conclusion is that it is urgent to rethink the prevailing training to open it towards an integrative inter and transdisciplinary pedagogical conception, and to improve the presence of sciences on the news agenda.

Keywords: communication of sciences; scientific journalism; specialized journalism; journalistic training; integrating higher education.

Copyright: () 2022 CSIC. Este es un artículo de acceso abierto distribuido bajo los términos de la licencia de uso y distribución Creative Commons Reconocimiento 4.0 Internacional (CC BY 4.0). 


\section{INTRODUCCIÓN}

En la tradición académica española, los estudios de Periodismo se encuadran en las Facultades de Ciencias Sociales y de Humanidades. Los alumnos que acceden a las titulaciones impartidas por estos centros proceden, en su mayoría, del Bachillerato de Ciencias Sociales. Esta circunstancia junto con el hecho de que los medios informativos deben afrontar la responsabilidad de informar a la sociedad de asuntos científico-tecnológicos cada vez más abundantes, notorios y determinantes, a la par que complejos, hace que nos cuestionemos sobre su autopercepción respecto al periodismo centrado en temáticas científicas. Para saberlo partimos de una dicotomía existente en el sistema académico español -incluso en la sociedad española- que opone Ciencias y Letras.

Es sabido que la enseñanza superior en España, desde la implantación de los estudios de Grado adaptados al Espacio Europeo de Educación Superior (EEES) (Ministerio de Educación y Ciencia, 2007) incorporó en los planes de estudios un cierto nivel de integración de disciplinas de la rama del conocimiento propia y de otras (Ramírez y Clemente, 2020). No obstante, esa integración, organizada en un principio en torno a las asignaturas de formación básica ${ }^{1}$ impartidas en los dos primeros cursos de la carrera, viene afrontando la dificultad de adaptarse a un aprendizaje integrado inter y transdisciplinar. En otros casos incide en la integración de las tecnologías (inteligencia artificial, robots) con uso en el periodismo transmedia ligado a la convergencia de medios en Europa (IJIE, 2014).

A esta realidad se une que los estudiantes ingresan en Periodismo con unas preferencias académicas y profesionales definidas a lo largo del Bachillerato. La estructura de este nivel educativo nos hace preguntarnos si esas preferencias, por lo común estructuradas en torno a la dicotomía ciencias/letras, se modifican a lo largo de la carrera y si van definiéndose perfiles orientados a trabajar en periodismo científico.

Consideramos que el periodismo se encuentra ante una situación compleja y desafiante que exige repensar el periodismo especializado en temas científicos, tecnológicos y medioambientales desde otras perspectivas formativas innovadoras. El contexto actual ofrece innumerables ejemplos en los que científicos y tecnólogos realizan, de forma más o menos continuada, tareas de comunicación social de sus disciplinas, sin requerir mediación periodística $y$, según encuestas realizadas en Europa, con mayor aceptación y confianza del público frente a los periodistas (Claessens, 2008). De hecho, los suplementos de medios informativos de referencia en España (El País y Prensa Ibérica) han incorporado a sus redacciones webs surgidas externamente a ellos de la mano de jóvenes universitarios licenciados en titulaciones englobadas en las STEMM (siglas de las disciplinas Science, Technology, Engineering, Mathematics y Medicine) y en Humanidades; es decir, por equipos humanos con formación diferente a Comunicación y Periodismo.

\subsection{Referentes contextuales}

La formación en Periodismo sigue siendo necesaria porque instruye en aspectos fundamentales de la profesión como, por ejemplo, el estilo, los géneros, los métodos de trabajo periodísticos, la historia y las teorías sociales, y, subrayémoslo, en la ética y deontología periodísticas. Este aprendizaje faculta para ofrecer un periodismo garante de los derechos relacionados con la información en las sociedades democráticas. Observando esta formación y la dinámica de la sociedad actual, coincidimos con autores como Fernández (2013) que defiende formar en ciencias a los estudiantes de humanidades para que puedan participar con criterio en los principales debates de la sociedad. Es una situación que plantea la conveniencia de dar un giro en la concepción de estos estudios en el panorama universitario español con el fin de sumar fuerzas e intereses académicos y profesionales de los universitarios de las STEMM a las propias de los que estudian Humanidades y Sociales (HS), y viceversa.

En la línea de lo indicado arriba, desde el punto de vista formativo es útil conocer la percepción académica y profesional que los estudiantes de Comunicación Social (Grados de Periodismo y de Comunicación Audiovisual) tienen de los asuntos científicos como materia prima periodística. Subyace el propósito de reflexionar sobre los estudios universitarios de Periodismo en España como una propuesta transversal a las distintas ramas de conocimiento, es decir, como una formación integradora ofrecida a los alumnos de los distintos campus de las universidades españolas (Ciencias, Ingenierías, Salud, Artes y Humanidades, Sociales y Jurídicas). Es una idea en cierto sentido rompedora con la tradición académica española que considera los estudios de Periodismo (desde la implantación de la Licenciatura anterior) insertos en el campus de Ciencias Sociales o en el de Humanidades, dependiendo de la universidad. Por lo que habría que sondear la posibilidad de concebirlos de una manera alternativa a esta exclusivamente unidisciplinaria.

El procedimiento seguido para diseñar y acreditar las titulaciones de Grado en España implica 
varios condicionantes; no obstante, consideramos que la idiosincrasia de estos estudios y de la profesión periodística sobrepasa estas barreras, ya que cada vez se admite que se requiere incorporar contenidos teórico-prácticos originados en otras áreas de conocimiento, sin que ello desnaturalice, bien al contrario, la formación en Periodismo. La idea de ofrecer titulaciones conjuntas con otras Facultades y Departamentos universitarios -por ejemplo, de las STEMM- es defendida por la Carnegie-Knight Initiative on the Future of Journalism Education; según Hume (2007), los integrantes de esta institución sustentan y ejemplifican esta propuesta en que permite a los futuros periodistas formarse y comprender los temas de esas disciplinas para cubrirlos mejor, y porque genera más respeto por los estudios de periodismo en el mundo académico.

Es la idea de una formación universitaria integrada, defendida e impulsada por The National Academies of Sciences, Engineering, and Medicine (2018), y sostenida ya antes por Wilson (1999), desde su perspectiva consiliente del conocimiento científico, y por Gaita (2012), con su visión crítica de la formación universitaria compartimentada y su enfoque que reta a comprender los descubrimientos de las ciencias naturales desde las humanidades. El análisis de la Academia estadounidense tiene en cuenta las barreras universitarias para la integración curricular, como, por ejemplo, la estructura y el funcionamiento de los Departamentos, similares a las que podríamos alegar en España; pero es a la vez optimista como muestran las decenas de titulaciones integradas impartidas por las universidades de EE.UU. que menciona.

Como afirman Barel-Ben, y otros (2020), el riesgo que afronta el periodismo es verse relegado a informar de la actualidad en sentido estricto, sobre todo de política y de otros asuntos ligeros, curiosidades o personajes famosos y celebridades. Sería la antesala de ver el periodismo de ciencias ejercido por graduados en esos campos del saber, en los que vienen demostrando sus habilidades para comunicar al público asuntos científicos más o menos complejos, interesantes e importantes de forma clara, comprometida, útil y entretenida. Son graduados en titulaciones distintas a la de Periodismo para quienes la comunicación pública es una salida o un complemento profesional con el perfil de redactores científicos en medios periodísticos, pero sin mediación de periodistas. Otro escenario estudiado por Peters (2014) lo dibujan los científicos que desarrollan actividades de difusión de las ciencias de manera directa con el público. No se trata, por tanto, de acciones divulgativas esporádicas. Hoy los periodistas conviven, como señala Miller (2008), con otros agentes de difusión sin forma- ción comunicativa ni periodística, con procedencia y objetivos diversos.

La revisión bibliográfica realizada por Barel-Ben y colaboradores (2020) deja constancia de la escasez de periodistas científicos a nivel internacional. $\mathrm{Su}$ estudio muestra los resultados positivos obtenidos de la lectura de textos dedicados a temas de ciencias escritos por científicos y publicados por medios informativos digitales israelíes; los autores constatan que estos textos son tan leídos como los redactados por periodistas sobre otros temas y publicados en la misma fecha que aquellos. A la vez inciden en la preparación de los periodistas para redactar de manera atractiva, entretenida e interesante. Planteamos si nos encontramos ante dos salidas: la colaboración entre ambos perfiles académico-profesionales para garantizar un periodismo científico de calidad, y la adaptación de la formación en periodismo científico a las exigencias de una sociedad dependiente de las ciencias y las tecnologías.

En el caso español, con vistas a fomentar el número de periodistas dedicados a informar de las ciencias, se presentan tres vías; una de ellas es prolongar la situación actual; otra es la vía colaborativa, que incide en fomentar la coautoría de periodistas y expertos con el reconocimiento académico y profesional para estos (Barel-Ben y otros, 2020; Meneses y Rivero, 2017; Riedlinger y otros, 2019); y la tercera vía apuesta por innovar de forma rompedora en los estudios de Grado en Periodismo, abriéndolos a estudiantes de las distintas ramas de conocimiento. La segunda y la tercera vía aprovecharían la formación dominante en cada grupo de profesionales (periodistas y científicos). Además, está la opción drástica de confiar a científicos la elaboración de estos contenidos; en este caso se mostraría que los periodistas son prescindibles, y para las audiencias se comprometerían las garantías éticas, deontológicas, normativas, metodológicas y de contrapoder en las que se forman los periodistas.

Para estimar la validez de cada opción resulta útil analizar la autopercepción de los estudiantes de Comunicación Social respecto a dedicarse a informar de estos temas frente a otros asuntos considerados tradicionalmente de letras (cultura, política, sociedad). Esta reflexión parte del carácter insustituible de la mediación periodística en las sociedades modernas, sumamente tecnologizadas y cada vez más dependientes de la gestión pública del conocimiento para mejorar la formación de los ciudadanos y neutralizar las no-noticias o fakenews. Que el público tome conocimiento de temas científicos gracias a los medios informativos le abre 
una ventana para interesarse por asuntos que contribuyen a mejorar su bienestar y calidad de vida.

En este estudio aplicamos la idea de ciencia en sentido amplio, desde luego no haciéndola depender de una rama de conocimiento concreta, sino de todas. $Y$ reflexionamos en torno a otra segunda idea clave: la integración disciplinar en la formación de los estudiantes de Comunicación, es decir, exponemos la posibilidad de considerarla una titulación transversal a las distintas ramas de conocimiento $y$, por tanto, a los distintos campus en los que se estructuran las universidades españolas.

\section{ESTADO DE LA CUESTIÓN}

Nuestra revisión bibliográfica no aporta estudios sobre la autopercepción de los estudiantes de Periodismo respecto a la división ciencias/letras, al periodismo científico y a la comunicación social de la ciencia. Los autores definen dos grandes tendencias investigadoras. La primera de ellas se centra en la formación periodística -en la que se encuadra el presente estudio-, en el análisis de contenido, en las fuentes y en la audiencia. La segunda tendencia se interesa por las dos culturas de Charles P. Snow, en el debate surgido en torno a la tercera cultura y su relación con la comunicación social de la ciencia.

Ejemplificamos la primera tendencia con Eide y Ottosen (1994), que señalan la escasez de periodistas científicos y de formación específica en Noruega; Dunwoody $(2004,2014)$ y Menezes (2018), que analizan el perfil de los periodistas científicos en EE.UU., como también hacen Polman y otros (2012), defensores de aplicar la práctica del periodismo científico con estudiantes de instituto para ayudarles a ser ciudadanos formados e informados sobre ciencias; Metcalfe y Gascoigne (1995) y Furlan (2016), que tras estudiar la situación en Australia concluyen que las habilidades periodísticas por sí solas son insuficientes para interpretar temas complejos de ciencia y medicina; Vernal y otros. (2019), que repasan la formación en ciencia y tecnología de los periodistas chilenos, valorándola como escasa, tal y como lo hiciera Dellamea (2000) desde los años 90 para Argentina, Benetti (2005), Brandão (2009) y Dos Santos (2012) para Brasil, y Hermelin (2011) para Colombia; Moreno y Gómez (2002), que sintetizan la formación en periodismo científico en España antes de la implantación de los Grados y las iniciativas internacionales surgidas desde los años setenta; Meneses y Rivero (2017), que revisan las asignaturas relacionadas con periodismo de ciencia, tecnología y medioambiente impartidas en los Grados de Periodismo y Comunicación Social en España, desde la perspec- tiva del sistema I+D+i; y Cassany y otros (2018), que muestran la formación dispar de los periodistas científicos españoles.

Y entre los investigadores de la segunda tendencia cabe mencionar a Brockman (1996), Llopis (2008) y Fernández (2013). Se suman otros enfoques investigadores como los ocupados de los modelos de comunicación científica, de la percepción social de la ciencia, y de los procesos y métodos de trabajo de los periodistas.

Estos autores australianos, canadienses, estadounidenses, europeos, iberoamericanos e israelíes muestran un panorama con un denominador común: la escasez de periodistas científicos y la demanda de más formación en periodismo científico. $Y$ coinciden en que el periodista de ciencias debe poseer una preparación que complemente la formación sobre teorías de la comunicación, estilo y redacción periodística, modalidades de periodismo, ética y deontología profesionales e historia del periodismo. Por lo que el reto es averiguar la razón de este déficit de periodismo y periodistas científicos, y cómo paliarlo en las aulas universitarias.

\section{OBJETIVOS}

El objeto de este trabajo fue conocer la percepción que tienen los estudiantes de Comunicación Social (Periodismo y Comunicación Audiovisual) sobre la dicotomía ciencias/letras. Partimos de los objetivos siguientes:

O1. Conocer cuál es la idea de ciencia que tienen los estudiantes de Comunicación Social y si la asocian a determinadas disciplinas.

O2. Averiguar si los estudiantes de Comunicación Social conciben la carrera que cursan como de ciencias, de letras o transversal a ambas.

O3. Evidenciar si la percepción que tienen los estudiantes de Comunicación Social sobre la ciencia varía a lo largo de los estudios de Grado.

\section{MATERIAL Y MÉTODO}

Planteamos una investigación descriptiva y transversal para conocer la presencia del fenómeno dicotómico ciencias/letras en estudiantes universitarios de Comunicación Social. Configuramos una muestra intencional entre estudiantes de la Universidad de La Laguna (ULL) y de la Universidad Complutense de Madrid (UCM) a los que encuestamos de manera presencial, anónima y mediante cuestionarios impresos. Obtuvimos datos de 327 estudiantes: 159 de Periodismo de la ULL (único grado de Comunicación impartido), y 92 de Periodismo de la UCM y 76 de Comunicación Audiovisual de la UCM (Tabla I), ya que tuvimos en cuenta que 
Tabla I. Participantes según Universidad y curso

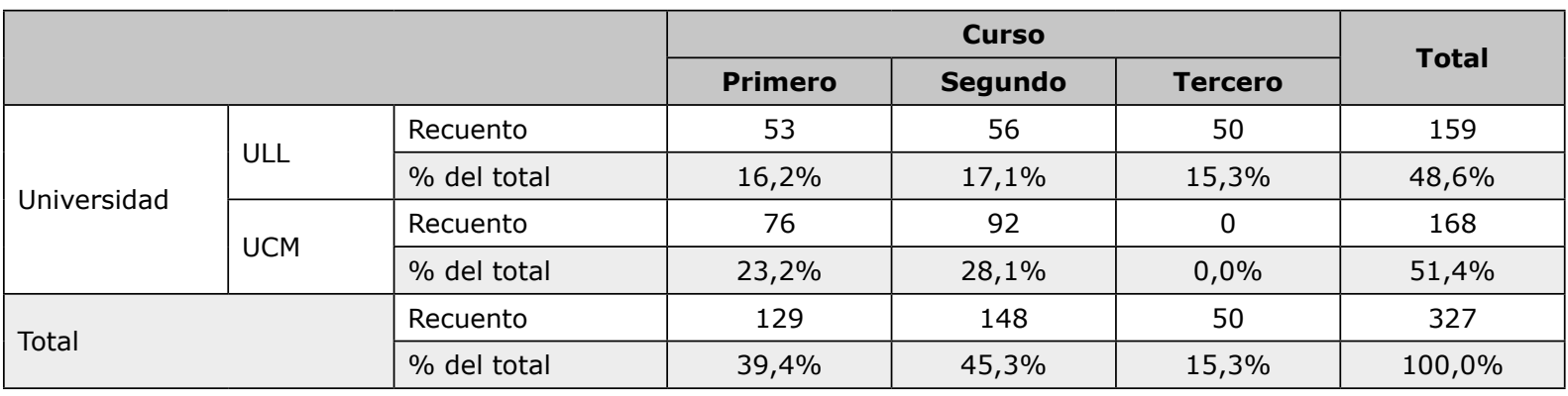

en España pueden ejercer el periodismo graduados de ambas titulaciones.

Diseñamos un cuestionario estructurado en nueve preguntas abiertas, dicotómicas y en escala. La primera pregunta fue de tipo dicotómico: '¿Te consideras un universitario de ciencias o de letras?', donde las opciones de respuestas eran 'ciencias', 'letras' y 'otra opción'. Así, nos acercamos a la posición de los participantes sobre la idea binaria del conocimiento y de la investigación científica. En la segunda, de respuesta abierta, pedimos que citaran cinco disciplinas que considerasen científicas para conocer la idea de ciencia de los participantes. En las dos preguntas siguientes planteamos dos bloques en los que debían responder si se consideraban estudiantes más de letras (B1) o más de ciencias (B2), ofreciéndoles seis respuestas que debían puntuar de 1 al 5 según el grado de acuerdo con cada una, siendo 1 el grado mínimo de acuerdo y 5 el máximo. Ambos bloques permitieron averiguar por qué se consideraban estudiantes de una u otra opción, debiendo puntuar si se consideran de letras o de ciencias porque las ciencias o las letras:

- No me gustan.

- No se me dan bien.

- No me las explicaron de forma amena y comprensible en Primaria, ESO o Bachillerato.

- Son difíciles.

- Conozco más las letras por mi ambiente familiar [si elegían la opción 1] / conozco más las ciencias por mi ambiente familiar [si elegían la opción 2].

- Por otra razón, ¿cuál?

Estas seis respuestas estaban presentadas en el mismo orden en cada bloque.

La quinta pregunta, también de tipo dicotómico, se enfocaba hacia la visión que tienen los participantes de los profesionales de las STEMM y de las HS: 'Marca con una $X$ lo que consideres que tiene más prestigio social: (elige solo una opción)'.

Mediante la sexta pregunta, '¿Quiénes consideras que son los intelectuales de una sociedad? (elige solo una opción)', buscamos conocer la percepción de los estudiantes sobre los profesionales académicos, según la dicotomía ciencias/letras.

La pregunta siguiente, de tipo dicotómico, pedía situar la carrera de Periodismo como de 'ciencias', 'letras', 'las dos (ciencias y letras)' o 'ninguna de las dos'. El objetivo era observar si perciben o no esta disciplina como transversal e integradora. En caso de marcar la última opción, se daba un espacio para especificar libremente qué tipo de carrera considera que es Periodismo.

La octava pregunta, abierta, vuelve a centrarse en la idea de ciencia de los participantes, aunque esta vez desde lo que observan en los medios de comunicación: '¿Qué materia o asunto consideras que es tratado en las noticias como científico por excelencia?'

El cuestionario termina con una pregunta dicotómica que insiste en la idea binaria que separa las STEMM y las HS. 'Al margen de lo que has respondido más arriba, ¿consideras que la división entre ciencias y letras tiene sentido en nuestra sociedad?'

Los estudiantes contestaron el cuestionario de forma individual y anónima, en el aula de la Facultad correspondiente en cada curso y Universidad. Analizamos los datos con el programa informático SPSS. El procedimiento seguido fue la preparación de la base de datos y el volcado en ella de las respuestas de los cuestionarios. A continuación efectuamos el análisis estadístico descriptivo, representando en tablas y gráficas los resultados obtenidos. Asimismo, para comprobar las diferencias entre cursos y universidades aplicamos análisis de varianza (Anova), tablas de contingencia y análisis descriptivo.

\section{RESULTADOS}

Para analizar los resultados aportados por las respuestas, estructuramos las preguntas en tres grupos. El primero está compuesto por las preguntas 2 ('Cita cinco disciplinas que consideres 
representativas de tu idea de ciencia') y 7 ('Qué temática o asunto consideras que es tratado en las noticias como temática científica por excelencia'), que permitirán conocer cuál es la idea de ciencia de la población participante. El segundo grupo abarca las preguntas 1 ('Te consideras un universitario de...'), 3 ('Puntúa de 1 a 5, dando 1 a lo que menor peso das y 5 a lo que más, las afirmaciones siguientes. Elige solo un bloque'), 6 ('Consideras que Periodismo es una carrera de...') y 8 ('Al margen de lo que has respondido más arriba, ¿consideras que la división entre ciencias y letras tiene sentido en nuestra sociedad?'), es decir, todas las que inciden de forma directa sobre la dicotomía de ciencias/ letras. Como tercer grupo tuvimos en cuenta las preguntas 4 ('Marca con una $X$ lo que consideres que tiene más prestigio social') y 5 ('¿Quiénes consideras que son los intelectuales de una sociedad?'), que tratan sobre las cualidades atribuidas a los profesionales de las STEMM y las HS. Además, comparamos los resultados de ambas universidades. El Anexo reúne las respuestas obtenidas.

\subsection{Idea de ciencia de los encuestados}

Las respuestas a las preguntas 2 y 7 plasman la idea de ciencia de la población participante. Así, la pregunta 2, 'Cita cinco disciplinas que consideres representativas de tu idea de ciencia', arrojó respuestas muy variadas. Hubo 107 diferentes, aunque las más frecuentes fueron Biología, Física, Matemáticas y Química, todas propias de las STEMM. Son las únicas disciplinas que obtuvieron una frecuencia superior a 20 y que aparecieron en más del $40 \%$ de los cuestionarios de cada curso.
Por tanto, son las que más reflejan la visión de disciplina científica de los estudiantes de Periodismo y Comunicación Audiovisual.

Al agrupar todas las respuestas por ramas de conocimiento, observamos la diferencia entre las STEMM y las HS. Sumando las correspondientes a disciplinas de Ciencias, Ciencias de la Salud, Ingeniería y Arquitectura obtenemos un porcentaje del 77,5\%. Mientras que las disciplinas de Ciencias Sociales y Jurídicas y Arte y Humanidades suponen el $22,4 \%$ del total.

La distribución por cursos de las disciplinas solicitadas, y tras agruparlas por áreas de conocimiento, no muestra una evolución significativa del concepto de ciencia a lo largo del Grado (Tabla II y Figura 1). Practicamos la prueba del análisis de varianza, Anova, para comprobar si había un cambio significativo en las disciplinas mayoritarias escogidas por los estudiantes según su curso. Pero observamos que, a pesar de que esperábamos cierto cambio conforme se avanza en la formación universitaria, no se dio tal evolución académica.

La séptima pregunta, '¿Qué temática consideras que es tratada en las noticias como científica por excelencia?', también mostró respuestas muy variadas. Computamos las respuestas múltiples (con más de una temática), por lo que el total de respuestas $(\mathrm{N})$ para esta pregunta del cuestionario alcanzó la cifra de 415.

Las respuestas recogen 43 temáticas diferentes. De ellas, la única que se repite en cada curso con una frecuencia igual o superior a 10 es Medicina y

Tabla II. Distribución por curso de las ramas de conocimiento que los estudiantes relacionan con la idea de ciencia

\begin{tabular}{|c|c|c|c|c|c|c|c|c|}
\hline \multicolumn{9}{|c|}{ Tabulación cruzada, frecuencias y porcentajes } \\
\hline & & & \multicolumn{5}{|c|}{ Rama de conocimiento } & \multirow[b]{2}{*}{ Total } \\
\hline & & & Ciencias & $\begin{array}{l}\text { Ciencias de } \\
\text { la Salud }\end{array}$ & $\begin{array}{l}\text { Ingeniería y } \\
\text { Arquitectura }\end{array}$ & $\begin{array}{l}\text { Ciencias } \\
\text { Sociales }\end{array}$ & $\begin{array}{c}\text { Artes y } \\
\text { Humanidades }\end{array}$ & \\
\hline \multirow{6}{*}{ Curso } & \multirow[b]{2}{*}{10} & Recuento & 387 & 26 & 31 & 84 & 72 & 600 \\
\hline & & $\begin{array}{l}\% \text { de respuestas en } \\
\text { el curso }\end{array}$ & $64,5 \%$ & $4,3 \%$ & $5,2 \%$ & $14,0 \%$ & $12,0 \%$ & $100,0 \%$ \\
\hline & \multirow[b]{2}{*}{20} & Recuento & 468 & 35 & 46 & 72 & 54 & 675 \\
\hline & & $\begin{array}{l}\% \text { de respuestas en } \\
\text { el curso }\end{array}$ & $69,3 \%$ & $5,2 \%$ & $6,8 \%$ & $10,7 \%$ & $8,0 \%$ & $100,0 \%$ \\
\hline & \multirow[b]{2}{*}{30} & Recuento & 150 & 16 & 10 & 39 & 18 & 233 \\
\hline & & $\begin{array}{l}\text { \% de respuestas en } \\
\text { el curso }\end{array}$ & $64,4 \%$ & $6,9 \%$ & $4,3 \%$ & $16,7 \%$ & $7,7 \%$ & $100,0 \%$ \\
\hline \multirow{2}{*}{\multicolumn{2}{|c|}{ Total }} & Recuento & 1005 & 77 & 87 & 195 & 144 & 1508 \\
\hline & & $\%$ respecto al total & $66,6 \%$ & $5,1 \%$ & $5,8 \%$ & $12,9 \%$ & $9,5 \%$ & $100,0 \%$ \\
\hline
\end{tabular}

Nota: N (Recuento total) es mayor que la muestra (327) porque se permitían respuestas múltiples en varias preguntas. 
Figura 1. Distribución por curso de las ramas de conocimiento que los estudiantes relacionan con la idea de ciencia (Tabla II).

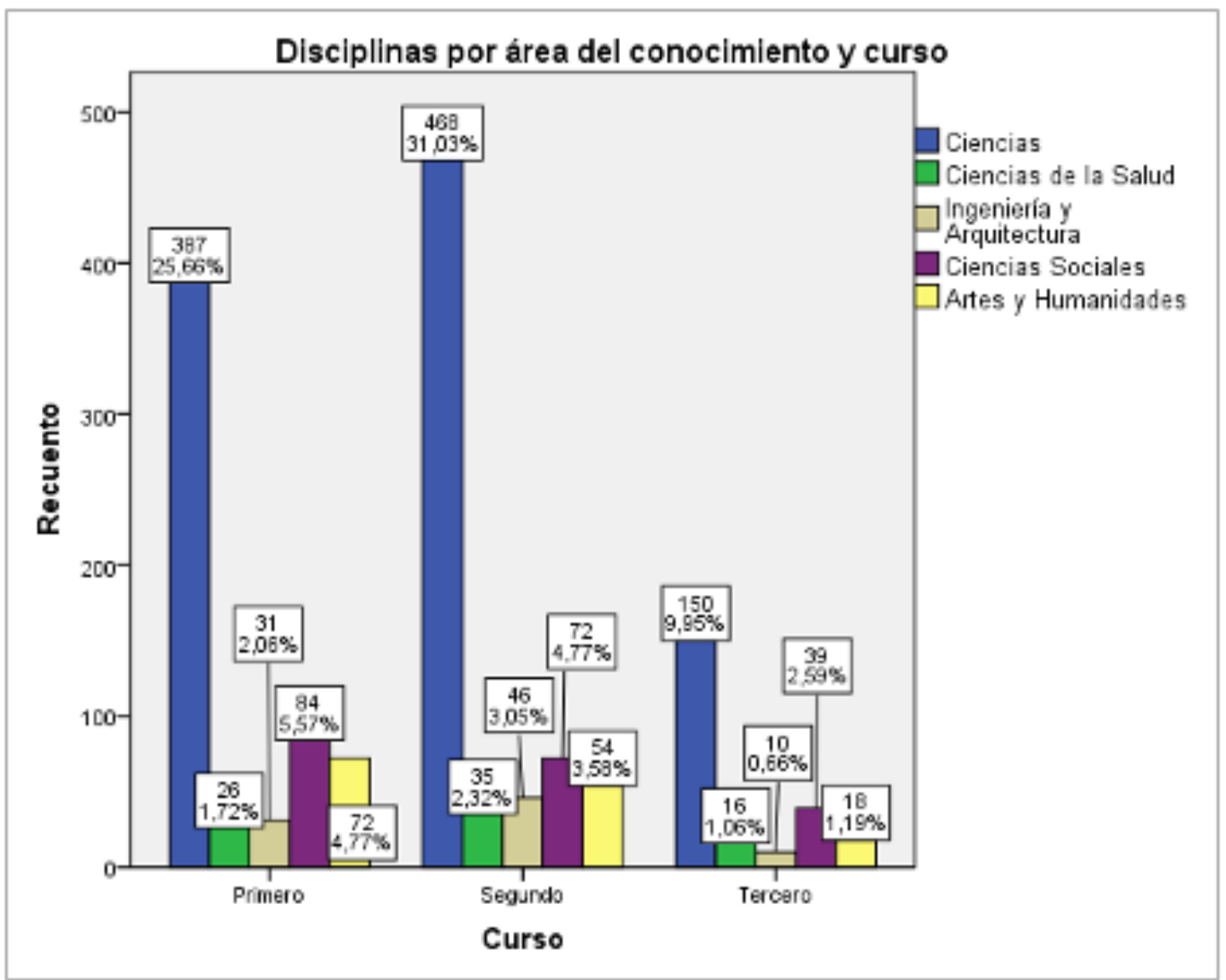

Salud. Otras que se repiten en más de un curso, pero no en todos, con frecuencias iguales o superiores a 10 fueron Tecnología, Desarrollos/Descubrimientos y Meteorología.

\subsection{Dicotomía ciencias/letras}

A la pregunta de elección simple '¿Te consideras un universitario de ciencias o de letras?', donde las opciones eran 'ciencias', 'letras' y 'otra opción', 247 $(76,5 \%)$ coinciden en la percepción de sí mismos como universitarios de letras. Mientras que solo 17 $(5,3 \%)$ se consideran universitarios de 'ciencias'. Por su parte, $59(18,3 \%)$ respondieron seleccionando 'otra opción'. La Figura 2 muestra los resultados a esta pregunta distribuidos por curso.

Solo 15 de los que escogieron 'otra opción' aprovecharon para reivindicar la Comunicación como una disciplina integradora y transversal; lo cual invita a reflexionar sobre la concepción de estos estudios superiores en la estructura tradicional por campus de las universidades españolas. De esta forma, si analizamos las respuestas alternativas ofrecidas por quienes marcaron 'otra opción' (Figura 2), se comprueba que la población participante utiliza esta tercera respuesta para identificarse con alguna rama del conocimiento, no para rehusar la dicotomía. Entre ellas destaca 'Ciencias Sociales' con el 39,7\% del total de respuestas alternativas. Otros afirman ser universitarios de 'Arte', de 'Audiovisuales', 'Humanidades' o 'Ciencias de la Información'.

En la tercera pregunta solicitamos a los participantes que respondieran solo a uno de los dos bloques incluidos en el cuestionario. Escoger el bloque 1 les situaba como estudiantes más 'de letras' y elegir el bloque 2 equivalía a considerarse más 'de ciencias'. En la línea de lo manifestado por los alumnos en la primera pregunta, $282(86,2 \%)$ 
Figura 2. Autopercepción respecto a la dicotomía ciencias/letras.

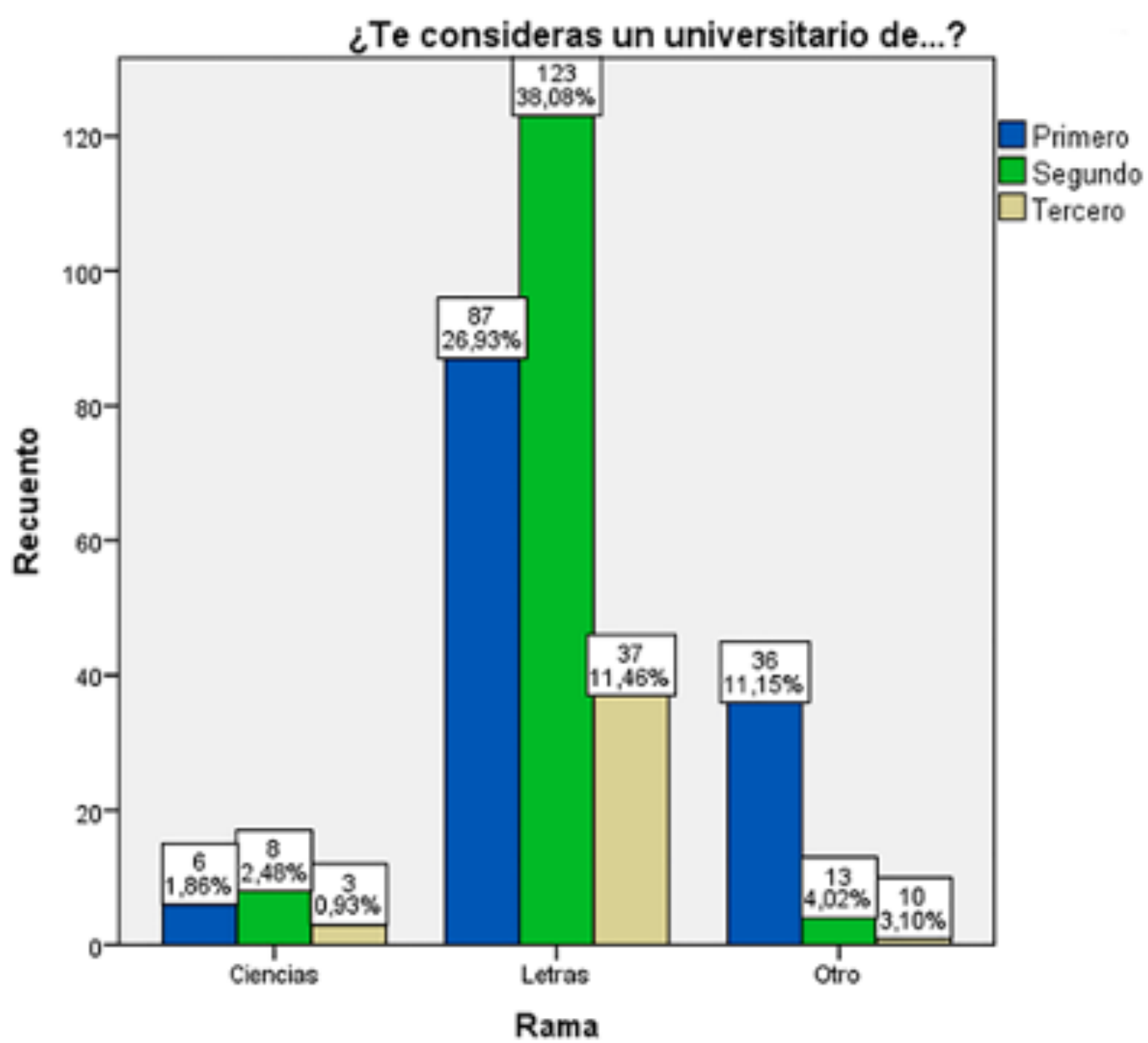

de ellos respondieron el bloque 1 , identificándose como estudiantes de letras. Mientras que 20 $(6,1 \%)$ contestaron el bloque 2 , definiéndose como estudiantes de ciencias.

En total (bloque de letras y bloque de ciencias) obtuvimos 302 respuestas válidas (92,4\%). Los 25 cuestionarios restantes hasta los 327 respondidos fueron descartados por saltarse el alumno el enunciado que le solicitaba elegir entre ambos bloques. De ellos, 5 no contestaron ninguno y 20 contestaron ambos, quedando los 25 invalidados. Cabría plantear si estos $20(6,1 \%)$ estudiantes podrían estar concibiendo el Grado que cursan como una formación transversal e integradora, no identificable con ciencias o con letras. Son respuestas que, de nuevo, sugieren reflexionar sobre si los estudios de Periodismo deben redimensionar su identidad, como titulación, al diseñar los programas curriculares.

Identificamos el grado de acuerdo de los participantes con cada una de las seis repuestas ofrecidas, aplicando estadística descriptiva. También tuvimos en cuenta la media, la moda y las medidas de dispersión (desviación estándar y varianza). Así, las respuestas del bloque 1 (de letras) muestran un nivel de acuerdo medio-bajo, donde la mayor concentración de respuestas se encuentra entre 1 (nada de acuerdo) y 3 (medianamente de acuerdo). El nivel de acuerdo varía ligeramente de una afirmación a otra con un rango de 0,49 sobre las medias y de 1,12 sobre las varianzas. Por tanto, ninguna de las afirmaciones planteadas explica por qué los alumnos se consideran de ciencias o de letras, aunque tampoco era el propósito de esta investigación. Esta vía queda abierta para resolverla en estudios futuros. Desechamos las respuestas del Bloque 2 (de ciencias) porque su bajo número (20) no permite garantizar la representatividad estadística.

En cuanto a referirse al Grado de Periodismo como una carrera de 'letras', de 'ciencias', de 'las dos (ciencias y letras)' o de 'ninguna de las dos', 192 la consideraron como de letras $(59,8 \%) ; 105$ 
Figura 3. Ubicación del Grado en Periodismo en la dicotomía ciencias/letras. Frecuencias por curso y porcentajes sobre el total de respuestas válidas $(\mathrm{N}=321)$. Línea discontinua: ' $X$. Línea continua: Me.

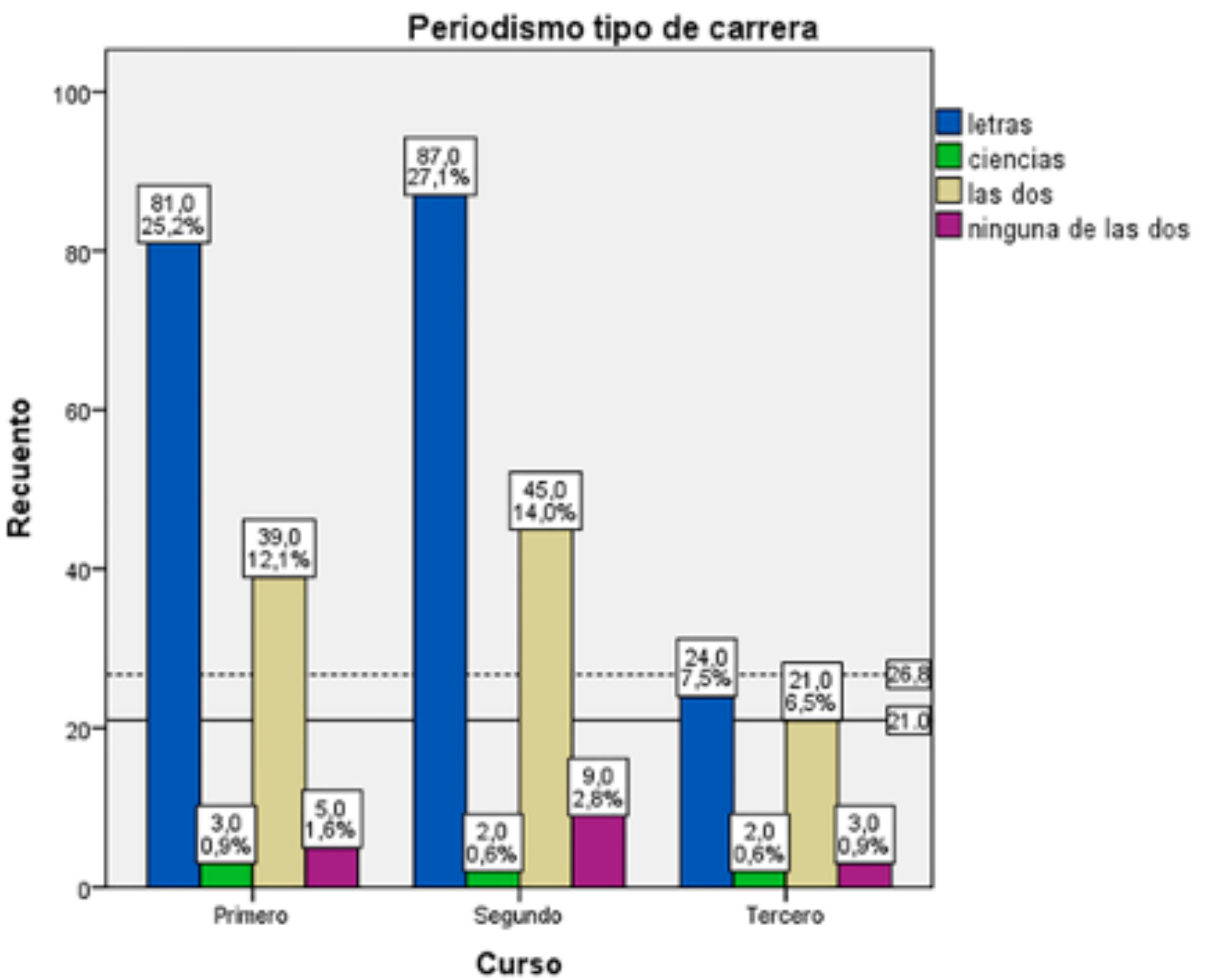

la consideran mixta de ciencias y letras $(32,7 \%)$; 17 como una ni de ciencias ni de letras $(5,3 \%)$ y 7 como una carrera de ciencias (2,2\%). Mostramos estos datos, agrupados por cursos, en la Figura 3.

Estas respuestas, sumadas a las de la primera pregunta y a las de los dos bloques, permiten confirmar que la mayoría de los estudiantes consideran que Periodismo es una carrera de letras. Finalmente, al pedirles que, al margen de lo respondido en el resto del cuestionario, dijeran si consideraban que la división entre ciencias y letras tiene sentido en nuestra sociedad respondieron conforme muestra la Figura 4. Si observamos las respuestas de forma global, sin separarlas por cursos, vemos que 182 alumnos $(55,7 \%)$ no consideran que tenga sentido la dicotomía de ciencias y letras. Aunque otros 135 (el $41,3 \%$ ) creen que sí lo tiene. Los 10 alumnos $(3,1 \%)$ restantes no contestaron esta pregunta.

Los porcentajes varían de forma considerable en el curso $3^{\circ}$, mientras que en $1^{\circ}$ y en $2^{\circ}$ son muy similares (Figura 4). Si atendemos a datos separados por cursos, los estudiantes de $1^{0}$ se mostraron conformes con la división ciencias/letras en un $44,2 \%$ de sus respuestas; mientras que el $55,0 \%$ no. Los de $2^{\circ}$ redujeron la diferencia entre ambas respuestas: marcaron sí el $45,9 \%$ de ellos y no el $48,6 \%$. Mientras que en 30 marcaron sí el $20,0 \%$ y el no aumenta hasta el $78,0 \%$. Este cambio permite pensar que determinadas asignaturas han podido influir en la percepción de los alumnos, favoreciendo su madurez académica y comprensión del verdadero sentido de la ciencia, de la investigación científica y de los conocimientos que genera.

\subsection{Cualidades de los profesionales de las STEMM y de las HS}

En este grupo analizamos las respuestas referidas a la percepción de las cualidades y prestigio de los profesionales de las ciencias y de las letras. Así, en la pregunta de selección simple 'Marca con una $X$ lo que consideres que tiene más prestigio so- 
Figura 4. Respuesta a la pregunta 'Al margen de lo que has respondido más arriba, ¿consideras que la división entre letras y ciencias tiene sentido en nuestra sociedad? (Elige solo una opción y márcala)'. Frecuencias por curso y porcentajes sobre el total de respuestas válidas. Línea discontinua: ‘ $X$. Línea continua: Me.

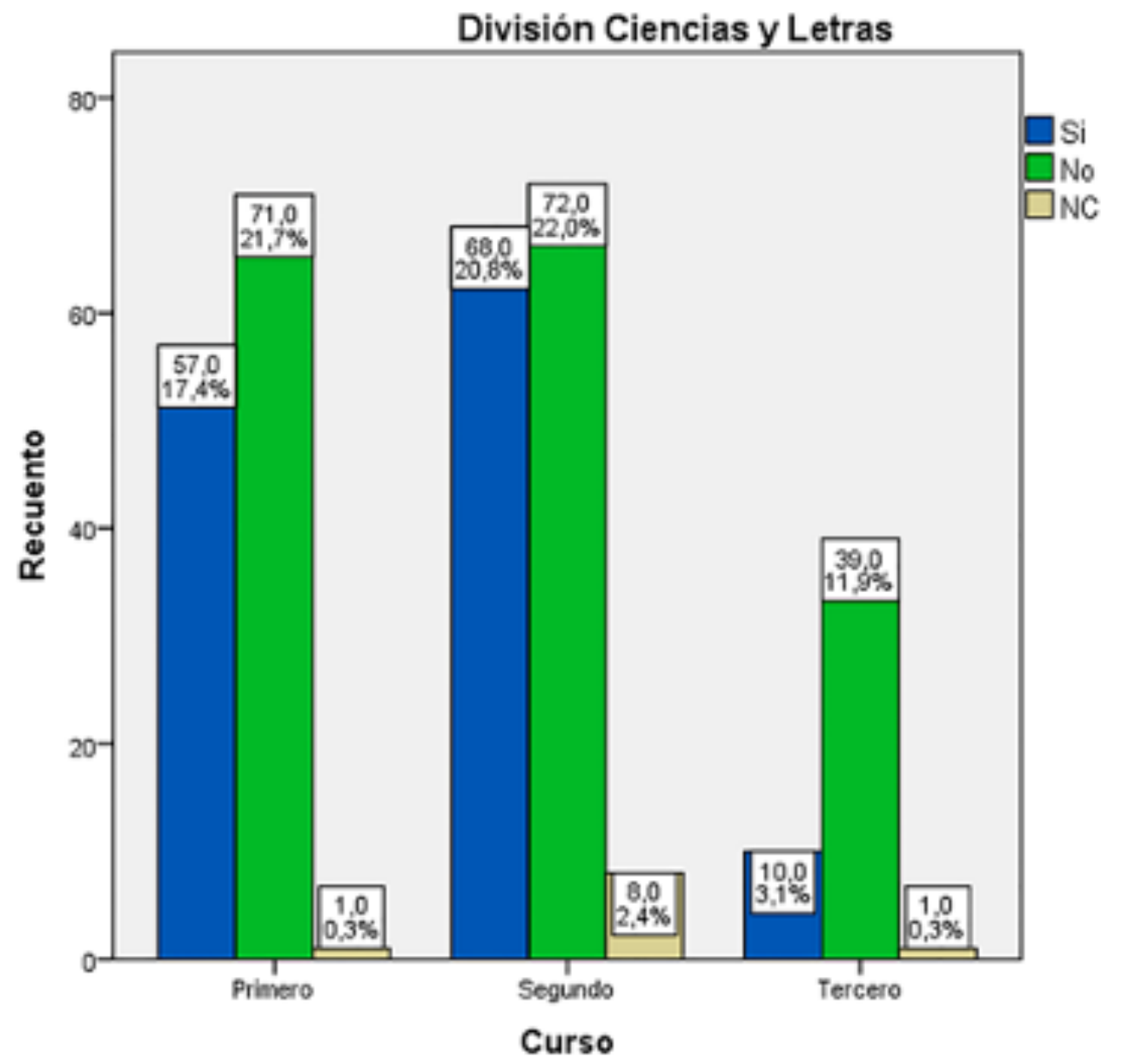

cial', la opción más elegida fue 'estudiar una carrera de ciencias' en 160 ocasiones $(49,7 \%)$, seguida de cerca por 'estudiar cualquiera de las 2 ' elegida en 151 ocasiones (46,9\%). La opción 'estudiar una carrera de letras' fue residual, ya que fue seleccionada solo por 11 alumnos (3,4\%).

En los tres cursos la opción más seleccionada fue la de 'una carrera de ciencias' (49,6\% en $10,49,7 \%$ en $2^{\circ}$ y $50,0 \%$ en $3^{\circ}$ ); la segunda fue 'cualquiera de las dos' $\left(48,8 \%\right.$ en $10,44,8 \%$ en $2^{\circ}$ y $48,0 \%$ en $\left.3^{\circ}\right)$; y la última, 'una carrera de letras' $(1,6 \%$ en $10,5,6 \%$ en 20 y $2,0 \%$ en $3^{\circ}$ ). Estas respuestas revelan una paradoja porque se desprenden dudas respecto a si los estudiantes de Comunicación Social consideran lo que están estudiando como disciplinas de prestigio, atributo reconocido a lo que posee cualidad o condición de científico.

Sin embargo, en la cuestión '¿Quiénes consideras que son los intelectuales de una sociedad?' se impone claramente la respuesta 'los dos por igual' con 235 respuestas $(73,2 \%)$. Sigue la opción 'los profesionales de las letras' seleccionada 37 veces $(11,5 \%)$. La tercera opción en orden decreciente fue la de 'los profesionales de las ciencias' con 25 $(7,8 \%)$. Por último, 'ninguna de las dos' fue seleccionada 24 veces $(7,5 \%)$.

La proporción de las valoraciones de los alumnos se mantiene sin grandes variaciones a lo largo de los tres cursos. Si bien en $3^{\circ}$ se iguala el número de alumnos que respondieron que los intelectuales de la sociedad son los 'profesionales de las ciencias' y los que respondieron los 'profesionales de las letras'.

\subsection{Comparación por universidades}

En el análisis Anova no se observan diferencias significativas entre las respuestas de los estudiantes de ambas universidades. En las preguntas de 
elección simple se detecta la mayor diferencia en la cuestión sobre el tipo de carrera que es Periodismo, que alcanza el 10,0\%. Esta está marcada por las respuestas de los alumnos de 10 de Comunicación Audiovisual de la UCM. Quizás porque identifican la palabra escrita y hablada como el código dominante mediante el que se trasladan los mensajes periodísticos, estableciendo por ello una relación directa con 'letras'.

De los 75 alumnos de $1^{\circ}$ de Comunicación Audiovisual, 54 (el $72 \%$ del curso) consideran que Periodismo es una carrera de letras; de los 91 estudiantes de $2^{\circ}$ de Periodismo de la UCM, 58 (el 63,7\% del curso) también entienden que es una carrera de letras. Sin embargo, los porcentajes en la ULL son menores en los tres cursos. En la clase de 10 , con 53 cuestionarios respondidos, 27 (50,9\%) creen que Periodismo es un Grado de letras; entre las 52 respuestas válidas de los alumnos de 20,29 $(55,8 \%)$ lo consideran de letras, y en 30 , de los 50 , $24(48 \%)$ lo describen como una carrera de letras.

En el resto de las preguntas de elección simple, las diferencias entre universidades son, en todos los casos, inferiores al $10 \%$.

\subsection{Discusión de los resultados}

Los periodistas formados en los Grados de Comunicación -en especial de Periodismo- deben saber desenvolverse, en su labor informativa, con conocimientos considerados en la tradición académica española de letras (por ejemplo, Historia, Lengua o Literatura) y de ciencias (por ejemplo, Astrofísica, Medicina o Medioambiente).

Resulta significativo que el $81,8 \%$ de alumnos asuman la dicotomía ciencias/letras y se muestren cómodos eligiendo una de estas dos etiquetas. Esta respuesta indica que la gran mayoría de los estudiantes encuestados se muestra conforme con esta idea binaria y excluyente que divide el conocimiento y la investigación científica entre ciencias y letras. Aunque preguntas posteriores del cuestionario nos permitieron observar si esta tendencia se mantiene o no.

Ante esa idea asumida, los resultados muestran que los estudiantes de Periodismo y de Comunicación Audiovisual se identifican a sí mismos como de letras. Se desmarcan de las STEMM, disciplinas a las que ven como algo ajeno a una titulación de Comunicación. Y observamos que, a lo largo del Grado, no varía su percepción de qué es ciencia.

Estas respuestas revelan que estos estudiantes perciben a los profesionales de las STEMM como más prestigiosos porque relacionan su trabajo con lo cuantitativo, lo preciso, y con el hecho de que los objetos con los que operan sean materiales y medibles, no etológicos ni simbólicos (Sokal y Brimont, 1999; Kreimer, 2010; Etcheverry, 2010; Gina, 2014; Rocke, 2014; Alvargonzález, 2019); además, como subrayan Gaita (2012) y Jacobs (2014), porque se muestran a los jóvenes con posibilidades profesionales e instrumentales distintas. Por ello podrían estar otorgando más reconocimiento a las STEMM respecto a las HS, que no vinculan con el marchamo de rigurosidad que otorgan lo cuantitativo y el método científico experimental. La formación que reciben estaría reforzándoles una concepción de noticia de ciencias escorada hacia las STEMM, en lugar de considerar, con sentido amplio e integrador, la investigación científica y la relevancia noticiosa de los avances producidos en las diversas ramas de conocimiento.

Como hemos expuesto, en distintos países es común el déficit de formación en periodismo científico y la escasa presencia en las redacciones de periodistas dedicados a temas científicos. Este hecho, junto con el distanciamiento de los temas científicos mostrado por el $67,60 \%$ de los alumnos de los Grados analizados (los que puntuaron con 5 que las ciencias no les gustan, no se les dan bien, no se las explicaron bien y son difíciles), pone en valor iniciativas innovadoras llevadas a cabo en universidades estadounidenses y francesas para formar en periodismo científico.

Orange y Turner (2013), Temple (2009) Thurman y otros (2016) describen un sector periodístico anglosajón que prioriza el componente de competitividad en el proceso de especialización. En Gran Bretaña pueden alcanzar la especialización cursando un programa formativo en Periodismo de dos años combinado o integrado en programas de licenciatura de tres años de otros ámbitos, por ejemplo, Ciencias (The University of Buckingham; University of Chester; University of Essex; London Metropolitan University; University of Westminster; University of Leeds ${ }^{2}$; Studyportals, 2020).

Junto con la opción anterior, las universidades británicas ofrecen estudios de tres años solo de Periodismo que incluyen formación opcional en Ciencia-Tecnología y Medioambiente, obteniendo la licenciatura en letras (Bachelor of Arts o B.A.). Y, además, en Gran Bretaña se puede estudiar Periodismo con nivel B.A., pero centrado en un aspecto temático concreto. Es el caso, por ejemplo, de la titulación Periodismo y Comunicación Medioambiental, impartida por la University of Brighton; aquí los estudiantes se forman en la gestión de riesgos naturales, el medio físico, la geografía y la geología, la geomática, los desastres naturales y la 
vulnerabilidad humana. De esta forma se da a los estudiantes la posibilidad de formarse en periodismo de ciencias y tecnologías desde el primer nivel universitario. Por tanto, en Gran Bretaña, los estudiantes disponen de vías diversas para convertir el periodismo de ciencias en una opción profesional.

Por su parte, Francia también ofrece iniciativas innovadoras y diversificadas. La tradición académica se combina con propuestas curriculares originales que promueven la interdisciplinariedad en la formación de los periodistas. En este país coexisten dos concepciones del periodismo: como un trabajo basado en una preparación técnica centrada en el saber hacer, impartida por las Écoles de Journalisme (Conférence Nationale des Métiers du Journalisme) y una duración de uno o dos años. $Y$ la otra vía son los estudios universitarios de Periodismo como los ofrecidos por la Sorbonne Université (París) que une Ciencias de la Vida y Tecnologías con Comunicación Social y Periodismo, en una licenciatura de tres años de su Faculté des Sciences et Ingénierie conjuntamente con la École Supérieure W. Esta formación académica se relaciona con la calidad periodística en temáticas científicas. Las exigencias académicas para la especialización hacen que cualquier periodista no sea considerado especializado (Marchetti, 2002, 2005).

Es en EE.UU. donde encontramos opciones renovadoras para ofrecer a los estudiantes estudios de Periodismo más vinculados a las ciencias. Su sistema universitario más abierto no encauza al alumno en un determinado ámbito como las titulaciones españolas. En los dos últimos años de las licenciaturas (Bachelor) los alumnos cursan asignaturas específicas de Periodismo, dedicadas a la redacción de noticias de áreas temáticas específicas, como información de ciencia, sanidad, agricultura, sistema alimentario y medioambiente, entre otras. Destacan las licenciaturas combinadas de Periodismo y otro campo disciplinar. Así, aunque las especializaciones sensu stricto se ofrecen en posgrados de periodismo de salud y medicina o de medioambiente y ciencia (v.g. Syracuse University; Arizona State University; University of Georgia; Columbia University; Universiy of Florida; University of North Carolina), la formación de Bachelor introduce cada vez más aspectos como cambio climático, sostenibilidad o comunicación de riesgos; o sobre métodos de trabajo, como el periodismo computacional y el reporterismo.

Un caso destacado es la Cornell University, cuyo Departamento de Comunicación imparte el programa de Grado de Comunicación de la Ciencia y Compromiso Público, con mención Bachelor of Science (licenciado en ciencias, en vez de B.A.), en el que trabaja Bruce V. Lewenstein, uno de los profesores de dicho Departamento y experto en comunicación pública de la ciencia. Son soluciones orientadas a paliar el déficit de periodismo científico detectado en los medios de comunicación por la escasa formación científica de los periodistas estadounidenses.

Las iniciativas puestas en marcha desde finales del siglo $X X$ en universidades estadounidenses se desarrollan en esta línea, basada en una formación superior concebida de manera integrada. Según The National Academies of Sciences, Engineering, and Medicine (2018), aplican la idea de integración desde la perspectiva curricular -contenidos, disciplinas en plural, visión inter y transdisciplinaria, métodos docentes, profesores, mundo profesional y empleadores-. Y la entienden como el diseño de una modalidad de aprendizaje y experiencia cognitiva de un estudiante que unifica diferentes enfoques disciplinarios, proyectada en cursos concretos o en planes de estudios (sobre enseñanza superior integrada véanse MacVicar, 1987; Herkert, 1997).

Este modelo es el que quizá subsanaría la percepción distante de las ciencias mostrada por los estudiantes de Comunicación Social encuestados. Es el que más promueve el periodismo de ciencia, tecnología y medioambiente, porque desde los primeros cursos del Grado permite ponerlos en contacto con los ámbitos disciplinares científicos y tecnológicos. De hecho, la UNESCO (2007; Banda, 2013) no considera los estudios de Periodismo una disciplina aislada, sino combinada con la enseñanza de las Humanidades y las Ciencias, y considera que los diseños formativos deberían articularse mediante la colaboración con docentes universitarios de humanidades y de ciencias. Es una visión académica que tiene en cuenta que el periodismo está en medio de todo lo que bulle en la sociedad, para cuya comprensión y traslación al público se debe formar a sus profesionales con esa perspectiva integradora. Este objetivo pasa por mejorar la percepción de las ciencias desde la etapa universitaria inicial.

\section{CONCLUSIONES}

Según lo expuesto, proponemos las conclusiones siguientes:

- Los datos revelan que los estudiantes de Periodismo y de Comunicación Audiovisual se consideran, por lo general, de letras. Así, el mayor número de ellos se identifica y sitúa en un lado de la dicotomía ciencias/letras. Esto podría influir en que excluyan de sus intereses profesionales informar de temas científicos, al considerarlos lejanos a la formación recibida. Téngase en cuenta que los resultados expuestos aquí muestran un alto porcentaje 
de estudiantes de comunicación que manifiestan no gustarles las ciencias, por lo que cabe cuestionarse qué predisposición mostrarán hacia el periodismo de ciencias. Es una cuestión que merece ser tratada en estudios futuros puesto que, además de ingresar en los grados de comunicación con unas preferencias definidas, no las modifican a lo largo de la carrera en favor de temas científicos. Por tanto, la dicotomía ciencias/letras es una separación que tienen asumida y que pocos de ellos cuestionan. De esta forma observamos que la idea de ciencia entre estos estudiantes es también dicotómica y la asocian, sobre todo, a disciplinas STEMM con las que, según sus respuestas, asocian el prestigio atribuido a lo científico (01). A la vez verificamos que la población participante considera que los Grados que cursa son carreras solo de letras (O2). Excepcionalmente la ven como una formación transversal a las distintas ramas de conocimiento. Esto nos conduce a plantear si un egresado de Comunicación pudiera no estar suficientemente preparado para comunicar sobre ciencias y tecnologías.

- La población participante no muestra una evolución significativa en su concepción de la idea de ciencias a lo largo de los tres primeros cursos de la carrera (03). A esta conclusión subyacen varias cuestiones que deberían llevar a reflexionar sobre qué visión de una sociedad dependiente de las ciencias y las tecnologías se les está inculcando durante su formación universitaria. A este respecto, consideramos que la dependencia formativa de másteres sobre comunicación de las ciencias actúa como un cuello de botella que incide en el carácter minoritario de los periodistas dedicados a informar de ellas.

- Otra conclusión, correlacionada con las anteriores, es que los estudiantes de Periodismo avanzan en los cursos sucesivos de la titulación sin llegar a considerarla científica, lo que genera dudas sobre cómo perciben la investigación realizada en Comunicación Social, en la que se sustenta su propia formación.

- Las diferencias detectadas en las respuestas de la población participante de las dos Universidades no son estadísticamente representativas. Según este resultado, proponemos considerar la autopercepción respecto a dicotomías ciencias/letras, mostrada en este trabajo, un rasgo común entre los estudiantes de Periodismo y Comunicación Audiovisual. No obstante, cabría extender el estudio a otras universidades para corroborar o matizar lo expuesto aquí.

- En su conjunto, las respuestas de los estudiantes revelan la conveniencia de ofrecer una formación periodística renovada y transversal, como ya vienen haciendo universidades de varios países.

Este trabajo lleva a reflexionar sobre alternativas para incentivar la comunicación periodística de las ciencias. Las propuestas de futuro deberán contemplar un replanteamiento audaz, y menos arraigado, de la formación de los periodistas para introducir un mayor nivel de integración disciplinar acorde con la complejidad del mundo actual. Es la línea seguida por las iniciativas citadas en los epígrafes Introducción y Estado de la Cuestión, y que vienen siendo aplicadas por cada vez más universidades mediante programas formativos transversales conjuntos. El propósito sería que los estudiantes se gradúen con una formación integrada y transversal que logre que las ciencias les resulten más cercanas para informar de todas ellas, dando sentido y significado a los avances de unas disciplinas desde la perspectiva de las otras.

\section{NOTAS}

1 Asignaturas que proporcionan al estudiante la formación básica estipulada en cada universidad. Pueden corresponder a la misma rama de conocimiento del Grado y a otras. En España suponen 60 ECTS de los 240 totales.

2 Las páginas webs de los portales académicos y de las universidades citadas fueron consultadas durante el curso académico 2019-2020.

\section{REFERENCIAS}

Alvargonzález, D. (2019). La clasificación de las ciencias desde la filosofía del cierre categorial. Revista de Humanidades, 37, 99-126. https://doi.org/10.5944/ rdh.37.2019.21202

Banda, F. (ed.) (2013). Model Curricula for Journalism Education. A Compendiun of New Syllabi. París: UNESCO.

Barel-Ben David, Y., Garty E.S., y Baram-Tsabari, A. (2020). Can scientists fill the science journalism void? Online public engagement with science stories authored by scientists. Plos One, 15(1), e0222250. https://doi.org/10.1371/journal.pone.0222250

Benetti Machado, M. (2005). Data and reflections on three journalism resarch environments. Brazilian Journalism Research, 1(1), 25-46. https://doi.org/10.25200/BJR. v1n1.2005.36

Brandão Tavares, F.M. (2009). O jornalismo especializado e a especialização periodística. Estudios em Comunicação, 5(Maio), 115-133.

Brockman, J. (1996). La tercera cultura. Más allá de la revolución científica. Barcelona: Tusquets.

Cassany, R., Cortiñas, S., y Elduque, A. (2018). Comunicar la ciencia: El perfil del periodista científico en España. Comunicar, XXVI(55), 9-18. https://doi.org/10.3916/ C55-2018-01.

Claessens, M. (2008). European Trends in Science Communication. En: Cheng, D., Claessens, M.. Gascoigne, T., Metcalfe, J., Schiele, B., y Shi, S. (eds.). Communicating Science in Social Contexts. New models, new practices, 27-38. European Commission: Springer. https://doi.org/10.1007/978-1-4020-8598-7_2 
Dellamea, A.B. (2000). La formación de divulgadores y periodistas científicos en la Argentina. En: Sociedad Mexicana para la Divulgación de la Ciencia y la Técnica. IX Congreso de Divulgación de la Ciencia y la Técnica. Morelia, Michoacán (México).

Dos Santos, M. (2012). O perfil de jornalistas na cobertura especializada em ciência [Profile of Brazilian journalists in covering science]. Comunicação \& Informação, 15(1), 197-216. http://dx.doi.org/10.5216/cei.v15i1.22510

Dunwoody, S. (2004). How valuable is formal science training to science journalists? Comunicação E Sociedade, 6, 7587. https://doi.org/10.17231/comsoc.6(2004).1229

Dunwoody, S. (2014). Science journalism. En: Bucchi, M., y Trench, B. (eds.). Handbook of Public Communication of Science and Technology, 15-26. New York: Routledge.

Etcheverry, G.J. (2010). ¿Dos culturas? O ninguna. Medicina (Buenos Aires), 70(5), 479-480.

Eide, M. y Ottosen, R. (1994). 'Science journalism' without science journalists: notes on a Norwegian media paradox. Public Understanding of Science, 3, 425-434. https://doi.org/10.1088/0963-6625/3/4/005

Fernández Buey, F. (2013). Para la tercera cultura. Ensayos sobre ciencias y humanidades. Barcelona: El Viejo Topo.

Furlan, P. (2016) Australian Medical/Health Journalists on the Value of Science-based Education and Training. Asia Pacific Media Educator, 26(2), 175-188. https:// doi.org/10.1177/1326365X16669194.

Gaita, R. (2012). To Civilise the City? Meanjin Quaterly, 71(1). Disponible en: https://meanjin.com.au/essays/ to-civilise-the-city/ [Fecha de consulta: 05/09/2018].

Gina (2014). ¿Estudias alguna ciencia social? iQue no te menosprecien! La Vanguardia (5 de junio). Disponible en: https://www.lavanguardia.com/cribeo/ geek/20140605/47314055496/estudias-algunaciencia-social-que-no-te-menosprecien.html [Fecha de consulta: 27/10/2018].

Hermelin, D. (2011). Un contexto para la comunicación pública de la ciencia y la tecnología en Colombia: de las herencias eurocéntricas a los modelos para la acción. Revista Co-herencia, 8(14), 231-260

Herkert, J.R. (1997). STS for engineers: Integrating Engineering, Humanities and Social Sciences. En: Annual Conference Proceedings, 15-15 Jun 1997. Milwaukee, Wisconsin (USA): ASEE. Disponible en: https://peer. asee.org/6799 [Fecha de consulta:11/07/2018]

Hume, E. (2007). University Journalism Education: A Global Challenge. A Report to the Center for International Media Assistance. Washington D.C.: CIMA-National Endowment for Democracy. Disponible en: https:// www.centerforinternationalmediaassistance.org/ wp-content/uploads/2015/02/CIMA-University Journalism_Education-Report.pdf [Fecha de consulta: 21/10/2018].

IJIE (2014). Integrated Journalism in Europe: Journalism Higher Education in Europe. Barcelona: IJIE Consortium. Disponible en: http://integratedjournalism.upf.edu/ wp-content/uploads/2015/06/Journalism-HigherEducation-in-Europe-DBbookJANUARY.pdf [Fecha de consulta: $17 / 10 / 2018]$.
Jacobs, P. (2014). A Major Milestone at Harvard Signals The Decline of Humanities. Business Insider. Disponible en: https://www.businessinsider.com/harvard-engineeringhumanities-2014-10?IR=T [Fecha de consulta: 02/08/2018].

Kreimer, P. (2010).¿Dos culturas o múltiples culturas? Ciencias duras, ciencias blandas y science studies. Medicina (Buenos Aires), 70(5), 475-478.

Llopis Goig, R. (2008). La 'tercera cultura' de Brockman. A distancia, 1, 169-175.

Marchetti, D. (2002). Les sous-champs spécialisés du journalisme. Réseaux, 2002/1(111), 22-55. Disponible en: https://www.cairn.info/revue-reseaux1-2002-1page-22.htm\# [Fecha de consulta: 19/01/2019].

Marchetti, D. (2005). Sub-fields of specialized journalism. En: Benson, R.; Neveu, E. (eds.), Bordieu and the Journalistic Field, 64-82. Cambridge (UK): Polity Press. https://doi.org/10.3917/res.111.0022

MacVicar, M.L.A. (1987). General Education for Scientists and Engineers: Current Issues and Challenges. Bulletin of Science, Technology \& Society, 7(5-6), 592-597. https://doi.org/10.1177/027046768700700329

Meneses Fernández, M.D., y Rivero Abreu, Y. (2017). La formación en periodismo científico desde la perspectiva del sistema nacional de I+D+i: el caso español. Cuadernos.Info, 41, 107-122. https://doi.org/10.7764/ cdi.41.1145

Menezes, S. (2018). Science Training for Journalists: An Essential Tool in the Post-Specialist Era of Journalism. Frontiers in Communication, 3, 1-5. https://doi. org/10.3389/fcomm.2018.00004.

Metcalfe, J.; Gascoigne, T. (1995). Science journalism in Australia. Public Understanding of Science, 4. 411428. https://doi.org/10.1088/0963-6625/4/4/005.

Miller, S. (2008). So Where's the Theory? on the Relationship between Science Communication Practice and Research. En: Cheng, D., Claessens, M., Gascoigne, T., Metcalfe, J., Schiele, B., y Shi, S. (eds.). Communicating Science in Social Contexts. New models, new practices, 275287. European Commission: Springer.

Ministerio de Educación y Ciencia (2007). Real Decreto $1393 / 2007$, de 29 de octubre, por el que se establece la ordenación de las enseñanzas universitarias oficiales. Disponible en: https://www.boe.es/eli/ es/rd/2007/10/29/1393/con [Fecha de consulta: 21/10/2018]

Moreno, C., y Gómez, J.L. (2002). Ciencia y tecnología en la formación de los futuros comunicadores. Comunicar, 19(octubre), 19-24.

National Academies of Sciences, Engineering and Medicine (2018). The Integration of the Humanities and Arts with Sciences, Engineering, and Medicine in Higher Education: Branches from the Same Tree. Washington, DC: The National Academies Press. https://doi. org/10.17226/24988

Peters, H.P. (2014). Las dos culturas. Científicos y periodistas, una relación todavía vigente. MÈTODE Science Studies Journal, 4, 163-169.

Polman, J.L., Newman, A., Farrar, C., y Saul, E.W. (2012). Science journalism: Students learn lifelong science literacy skills by reporting the news. The Science Teacher, 79 (1), 44- 47. 
Ramírez Alvarado, M.M., y Clemente Mediavilla, J. (2020). Docencia e Investigación en Comunicación en España. Salamanca: Asociación Española de Universidades con Titulaciones de Información y Comunicación. Disponible en: https://summa.upsa.es/viewer.vm?id=129385 [Fecha de consulta: 05/06/2020].

Riedlinger, M., Massarani, L., Joubert, M., Baram-Tsabari, A., Entradas, M., y Metcalfe, J. (2019). Telling stories in science communication: case studies of scholar-practitioner collaboration. Journal of Science Communication, 18(05), 1-14. https://doi.org/10.22323/2.18050801

Rocke, S. (2014). The Misery of Measurement: Humanities and the Loss of Mystery. Eras, 15 (March), 1-13. Disponible en: https://pdfs.semanticscholar.org/5fde/ e1c8440e1b7cff0391ef2c0fdb89f0abf45e.pdf $\quad$ [Fecha de consulta: 15/10/2018].

Sokal, A., y Bricmont, J. (1999). Imposturas intelectuales. Barcelona: Paidós [1998].

Temple, M. (2009). The Future of Journalism Education in the United Kingdom: a Personal View from the Academy. MediAnali, 3(5), 241-258. Disponible en: https://hrcak. srce.hr/file/61699 [Fecha de consulta: 16/10/2018].

Thurman, N., Cornia, A., y Kunert, J. (2016). Journalists In The UK. University of Oxford, Reuters Institute for the Study of Journalism. Disponible en: https://ora. ox.ac.uk/objects/uuid:5986a227-839c-4315-9ccc732b055a0461 [Fecha de consulta: 07/07/2018].

Turner, B., yOrange, R. (ed.)(2013). SpecialistJournalism. Londres: Routledge. https://doi.org/10.4324/9780203146644

UNESCO (2007). Plan modelo de estudios de periodismo. París: Sector de Comunicación e Información-UNESCO.

Vernal-Vilicic, T.P., Valderrama, L.B., Contreras-Ovalle, J., y Arriola, T. (2019). Percepción de la formación y la especialización del periodismo científico en Chile. Cuadernos.info, 45, 213-226. https://doi.org/10.7764/ cdi.45.1717.

Wilson, E.O. (1999). Consilience. La unidad del conocimiento. Barcelona: Galaxia Gutenberg.

\section{WEBS DE LAS UNIVERSIDADES Y ORGANIS- MOS DE ENSEÑANZA SUPERIOR CITADOS:}

Arizona State University (2020): https://www.asu.edu/

Columbia University: https://www.columbia.edu/

Cornell University: https://www.cornell.edu/

Conférence Nationale des Métiers du Journalisme: http:// www.cnmj.fr/basedocumentaire/ecoles-journalisme-reconnues/

EEES http://www.ehea.info/

École Supérieure W: https://www.ecolew.com/formation/ licence-sciences-communication-journalisme/

London Metropolitan University: https://www.londonmet. ac.uk/

Faculté des Sciences et Ingénierie de Sorbonne Université: https://sciences.sorbonne-universite.fr/licences/ doubles_cursus/sciences_communication_journalisme

Studyportals: https://studyportals.com/

Syracuse University: https://www.syracuse.edu/

The University of Buckingham: https://www.buckingham. ac.uk/

Universidad Nacional de Córdoba: https://www.unc.edu. ar/

Universidad Nacional de La Plata: https://unlp.edu.ar/ unlp

University of Chester: https://www1.chester.ac.uk/

University of Essex: https://www.essex.ac.uk/

University of Georgia: https://www.uga.edu/

University of Leeds: https://www.leeds.ac.uk/

University of North Carolina: https://www.unc.edu/

University of Westminster: https://www.westminster. ac.uk/

University of Florida: http://www.ufl.edu/ 


\section{ANEXO}

Tabla general con los datos correspondientes a los resultados derivados del procesamiento estadístico de las respuestas de la población participante.

Frecuencias absolutas (fi) y porcentajes (\%) de las cinco opciones de respuestas que los encuestados debían puntuar (P) con 1 (mínimo acuerdo), 2, 3, 4 o 5 (máximo acuerdo), B1: bloque letras, B2: bloque ciencias. Las celdas sin datos fueron opciones no seleccionadas por los participantes.

\begin{tabular}{|c|c|c|c|c|c|c|c|c|c|c|c|c|c|}
\hline \multirow{2}{*}{$\begin{array}{c}\text { Opciones de } \\
\text { respuesta } \\
\text { para el B1 }\end{array}$} & \multirow{2}{*}{$\mathbf{P}$} & & \multicolumn{3}{|c|}{ Curso } & \multirow{2}{*}{ Totales } & \multirow{2}{*}{$\begin{array}{c}\text { Opciones de } \\
\text { respuesta } \\
\text { para el B2 }\end{array}$} & \multirow{2}{*}{$\mathbf{P}$} & & \multicolumn{3}{|c|}{ Curso } & \multirow{2}{*}{ Totales } \\
\hline & & & 10 & 20 & $3^{\circ}$ & & & & & 10 & 20 & 30 & \\
\hline \multirow{10}{*}{ No me gustan } & \multirow[b]{2}{*}{1} & fi & 27 & 34 & 9 & 70 & \multirow{10}{*}{ No me gustan } & & \multirow{2}{*}{$1 \frac{f i}{o}$} & 2 & 2 & 5 & 9 \\
\hline & & $\%$ & 27,80 & 27,60 & 23,10 & 27,00 & & & & 28,60 & 100,00 & 83,30 & 60,00 \\
\hline & \multirow{2}{*}{2} & \multirow{2}{*}{$\begin{array}{l}\text { fi } \\
\%\end{array}$} & 14 & 24 & 11 & 49 & & \multirow{2}{*}{2} & fi & - & - & - & - \\
\hline & & & 14,40 & 19,50 & 28,20 & 18,90 & & & $\%$ & - & - & - & - \\
\hline & \multirow{2}{*}{3} & fi & 13 & 17 & 2 & 32 & & \multirow{2}{*}{3} & fi & - & - & - & - \\
\hline & & $\%$ & 13,40 & 13,80 & 5,10 & 12,40 & & & $\%$ & - & - & - & - \\
\hline & \multirow{2}{*}{4} & fi & 16 & 20 & 3 & 39 & & \multirow{2}{*}{4} & fi & 1 & 0 & 0 & 1 \\
\hline & & $\%$ & 16,50 & 16,30 & 7,70 & 15,10 & & & $\%$ & 14,30 & 0,00 & 0,00 & 6,70 \\
\hline & \multirow{2}{*}{5} & fi & 27 & 28 & 14 & 69 & & \multirow{2}{*}{5} & fi & 4 & 0 & 1 & 5 \\
\hline & & $\%$ & 27,80 & 22,80 & 35,90 & 26,60 & & & $\%$ & 57,10 & 0,00 & 16,70 & 33,30 \\
\hline \multirow{2}{*}{\multicolumn{2}{|c|}{ Totales }} & fi & 97 & 123 & 39 & 259 & \multirow{2}{*}{ Totales } & & fi & 7 & 2 & 6 & 15 \\
\hline & & $\%$ & 100,00 & 100,00 & 100,00 & 100,00 & & & $\%$ & 100,00 & 100,00 & 100,00 & 100,00 \\
\hline \multirow{10}{*}{$\begin{array}{l}\text { No se me dan } \\
\text { bien }\end{array}$} & \multirow{2}{*}{1} & fi & 18 & 21 & 5 & 44 & & \multirow{2}{*}{1} & fi & 3 & 1 & 2 & 6 \\
\hline & & $\%$ & 18,40 & 17,80 & 12,50 & 17,20 & & & $\%$ & 42,90 & 50,00 & 40,00 & 42,90 \\
\hline & 2 & fi & 24 & 34 & 5 & 63 & & 2 & fi & 1 & 1 & 1 & 3 \\
\hline & 2 & $\%$ & 24,50 & 28,80 & 12,50 & 24,60 & & & $\%$ & 14,30 & 50,00 & 20,00 & 21,40 \\
\hline & 3 & fi & 20 & 19 & 17 & 56 & No se me dan & & fi & 2 & 0 & 1 & 3 \\
\hline & נת & $\%$ & 20,40 & 16,10 & 42,50 & 21,90 & & | & $\%$ & 28,60 & 0,00 & 20,00 & 21,40 \\
\hline & 1 & fi & 21 & 25 & 10 & 56 & & 1 & fi & 1 & 0 & 1 & 2 \\
\hline & & $\%$ & 21,40 & 21,20 & 25,00 & 21,90 & & 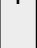 & $\%$ & 14,30 & 0,00 & 20,00 & 14,30 \\
\hline & 5 & fi & 15 & 19 & 3 & 37 & & 5 & fi & - & - & - & - \\
\hline & 3 & $\%$ & 15,30 & 16,10 & 7,50 & 14,50 & & $\rho^{3}$ & $\%$ & - & - & - & - \\
\hline Tat? & & fi & 98 & 118 & 40 & 256 & $T_{0}$ & & fi & 7 & 2 & 5 & 14 \\
\hline focarios & & $\%$ & 100,00 & 100,00 & 100,00 & 100,00 & perates & & $\%$ & 100,00 & 100,00 & 100,00 & 100,00 \\
\hline
\end{tabular}




\begin{tabular}{|c|c|c|c|c|c|c|c|c|c|c|c|c|c|}
\hline \multirow{2}{*}{$\begin{array}{c}\text { Opciones de } \\
\text { respuesta } \\
\text { para el B1 }\end{array}$} & \multirow{2}{*}{$\mathbf{P}$} & & \multicolumn{3}{|c|}{ Curso } & \multirow{2}{*}{ Totales } & \multirow{2}{*}{$\begin{array}{c}\text { Opciones de } \\
\text { respuesta } \\
\text { para el B2 }\end{array}$} & \multirow{2}{*}{$\mathbf{P}$} & & \multicolumn{3}{|c|}{ Curso } & \multirow{2}{*}{ Totales } \\
\hline & & & 10 & $2^{\circ}$ & 30 & & & & & $1^{\circ}$ & $2^{\circ}$ & $3^{\circ}$ & \\
\hline \multirow{10}{*}{$\begin{array}{l}\text { No me las } \\
\text { explicaron } \\
\text { de forma } \\
\text { amena y } \\
\text { comprensible }\end{array}$} & \multirow{2}{*}{1} & fi & 22 & 30 & 6 & 58 & \multirow{10}{*}{$\begin{array}{l}\text { No me las } \\
\text { explicaron } \\
\text { de forma } \\
\text { amena y } \\
\text { comprensible }\end{array}$} & \multirow{2}{*}{1} & fi & 2 & 1 & 1 & 4 \\
\hline & & $\%$ & 22,90 & 25,40 & 14,60 & 22,70 & & & $\%$ & 28,60 & 50,00 & 20,00 & 28,60 \\
\hline & \multirow{2}{*}{2} & fi & 19 & 17 & 7 & 43 & & \multirow{2}{*}{2} & fi & 1 & 0 & 1 & 2 \\
\hline & & $\%$ & 19,80 & 14,40 & 17,10 & 16,90 & & & $\%$ & 14,30 & 0,00 & 20,00 & 14,30 \\
\hline & \multirow{2}{*}{3} & fi & 23 & 31 & 12 & 66 & & \multirow{2}{*}{3} & fi & 2 & 0 & 1 & 3 \\
\hline & & $\%$ & 24,00 & 26,30 & 29,30 & 25,90 & & & $\%$ & 28,60 & 0,00 & 20,00 & 21,40 \\
\hline & \multirow{2}{*}{4} & fi & 14 & 20 & 9 & 43 & & \multirow{2}{*}{4} & fi & 2 & 1 & 2 & 5 \\
\hline & & $\%$ & 14,60 & 16,90 & 22,00 & 16,90 & & & $\%$ & 28,60 & 50,00 & 40,00 & 35,70 \\
\hline & \multirow{2}{*}{5} & fi & 18 & 20 & 7 & 45 & & \multirow{2}{*}{5} & fi & - & - & - & - \\
\hline & & $\%$ & 18,80 & 16,90 & 17,10 & 17,60 & & & $\%$ & - & - & - & - \\
\hline \multirow{2}{*}{\multicolumn{2}{|c|}{ Totales }} & fi & 96 & 118 & 41 & 255 & & & fi & 7 & 2 & 5 & 14 \\
\hline & & $\%$ & 100,00 & 100,00 & 100,00 & 100,00 & tolares & & $\%$ & 100,00 & 100,00 & 100,00 & 100,00 \\
\hline & & fi & 14 & 17 & 1 & 32 & & & fi & 2 & 1 & 1 & 4 \\
\hline & 1 & $\%$ & 14,70 & 14,40 & 2,50 & 12,60 & & 1 & $\%$ & 28,60 & 50,00 & 20,00 & 28,60 \\
\hline & 2 & fi & 25 & 23 & 16 & 64 & & 2 & fi & 3 & 0 & 3 & 6 \\
\hline & 2 & $\%$ & 26,30 & 19,50 & 40,00 & 25,30 & & $\left.\right|^{2}$ & $\%$ & 42,90 & 0,00 & 60,00 & 42,90 \\
\hline Con & & fi & 27 & 40 & 11 & 78 & & & fi & 1 & 1 & 0 & 2 \\
\hline Son dificiles & 3 & $\%$ & 28,40 & 33,90 & 27,50 & 30,80 & Son dificiles & 3 & $\%$ & 14,30 & 50,00 & 0,00 & 14,30 \\
\hline & 1 & fi & 16 & 28 & 9 & 53 & & 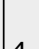 & fi & 1 & 0 & 1 & 2 \\
\hline & & $\%$ & 16,80 & 23,70 & 22,50 & 20,90 & & & $\%$ & 14,30 & 0,00 & 20,00 & 14,30 \\
\hline & & fi & 13 & 10 & 3 & 26 & & 5 & fi & - & - & - & - \\
\hline & J & $\%$ & 13,70 & 8,50 & 7,50 & 10,30 & & & $\%$ & - & - & - & - \\
\hline Totolos & & fi & 95 & 118 & 40 & 253 & Tatolo & & fi & 7 & 2 & 5 & 14 \\
\hline tolates & & $\%$ & 100,00 & 100,00 & 100,00 & 100,00 & tolates & & $\%$ & 100,00 & 100,00 & 100,00 & 100,00 \\
\hline & 1 & fi & 30 & 50 & 21 & 101 & & & fi & 1 & 0 & 2 & 3 \\
\hline & 1 & $\%$ & 31,90 & 44,60 & 55,30 & 41,40 & & 1 & $\%$ & 12,50 & 0,00 & 40,00 & 18,80 \\
\hline & & fi & 18 & 16 & 3 & 37 & & & fi & 1 & 1 & 0 & 2 \\
\hline & & $\%$ & 19,10 & 14,30 & 7,90 & 15,20 & & & $\%$ & 12,50 & 33,30 & 0,00 & 12,50 \\
\hline Ambiente & 3 & fi & 14 & 18 & 0 & 32 & Ambiente & 3 & fi & 2 & 0 & 0 & 2 \\
\hline familiar & 3 & $\%$ & 14,90 & 16,10 & 0,00 & 13,10 & familiar & 3 & $\%$ & 25,00 & 0,00 & 0,00 & 12,50 \\
\hline & & fi & 15 & 14 & 5 & 34 & & & fi & 1 & 0 & 1 & 2 \\
\hline & & $\%$ & 16,00 & 12,50 & 13,20 & 13,90 & & & $\%$ & 12,50 & 0,00 & 20,00 & 12,50 \\
\hline & 5 & fi & 17 & 14 & 9 & 40 & & & fi & 3 & 2 & 2 & 7 \\
\hline & & $\%$ & 18,10 & 12,50 & 23,70 & 16,40 & & 5 & $\%$ & 37,50 & 66,70 & 40,00 & 43,80 \\
\hline$T$ & & fi & 94 & 112 & 38 & 244 & & & fi & 8 & 3 & 5 & 16 \\
\hline & & $\%$ & 100,00 & 100,00 & 100,00 & 100,00 & & & $\%$ & 100,00 & 100,00 & 100,00 & 100,00 \\
\hline
\end{tabular}


Representación gráfica de los resultados correspondientes al bloque 1 de Letras: media de respuestas, según el curso, sobre los motivos por los que no les gustan las Ciencias.

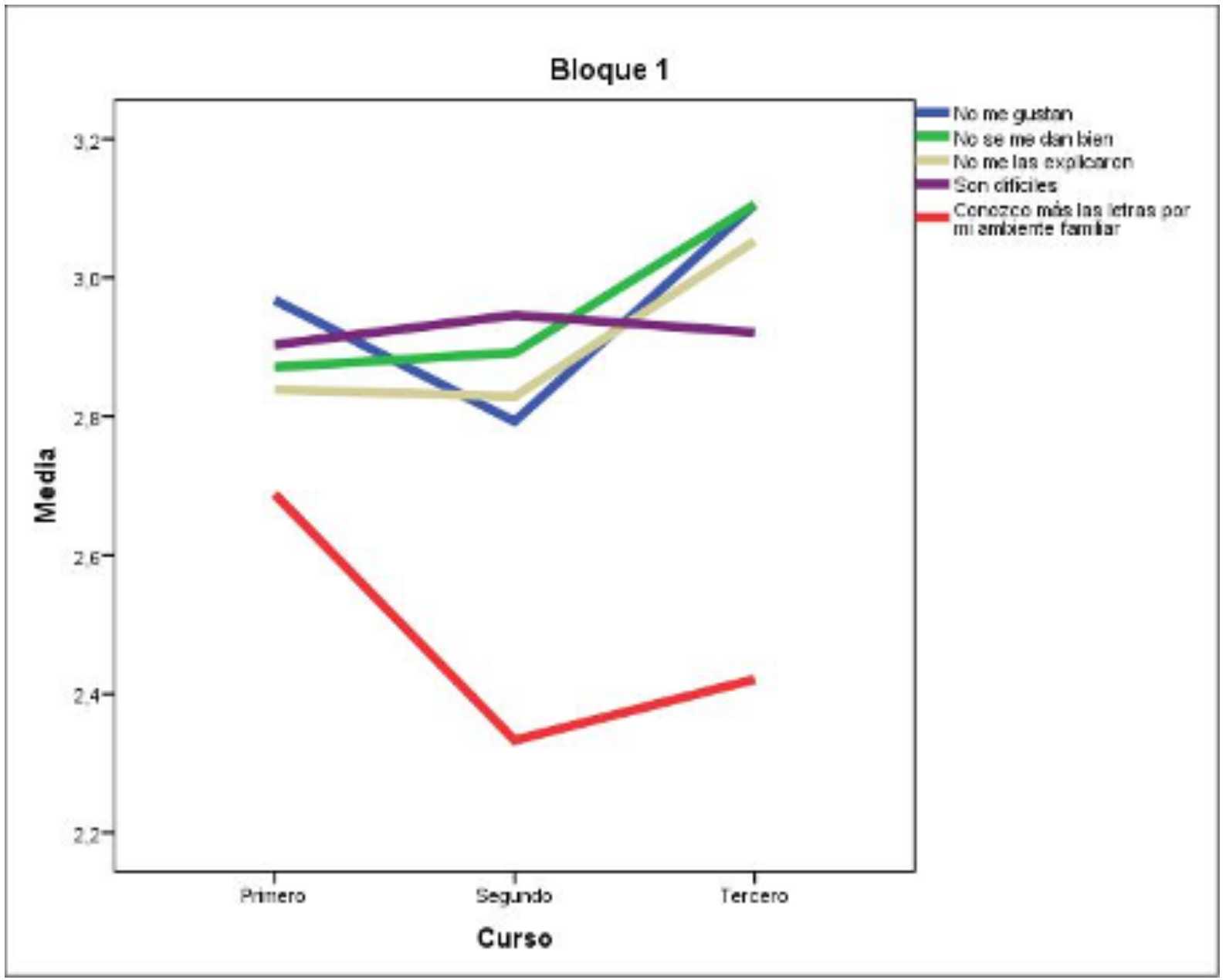


Representación gráfica de los resultados correspondientes al bloque 2 de Ciencias: media de respuestas, según el curso, sobre los motivos por los que no les gustan las Letras.

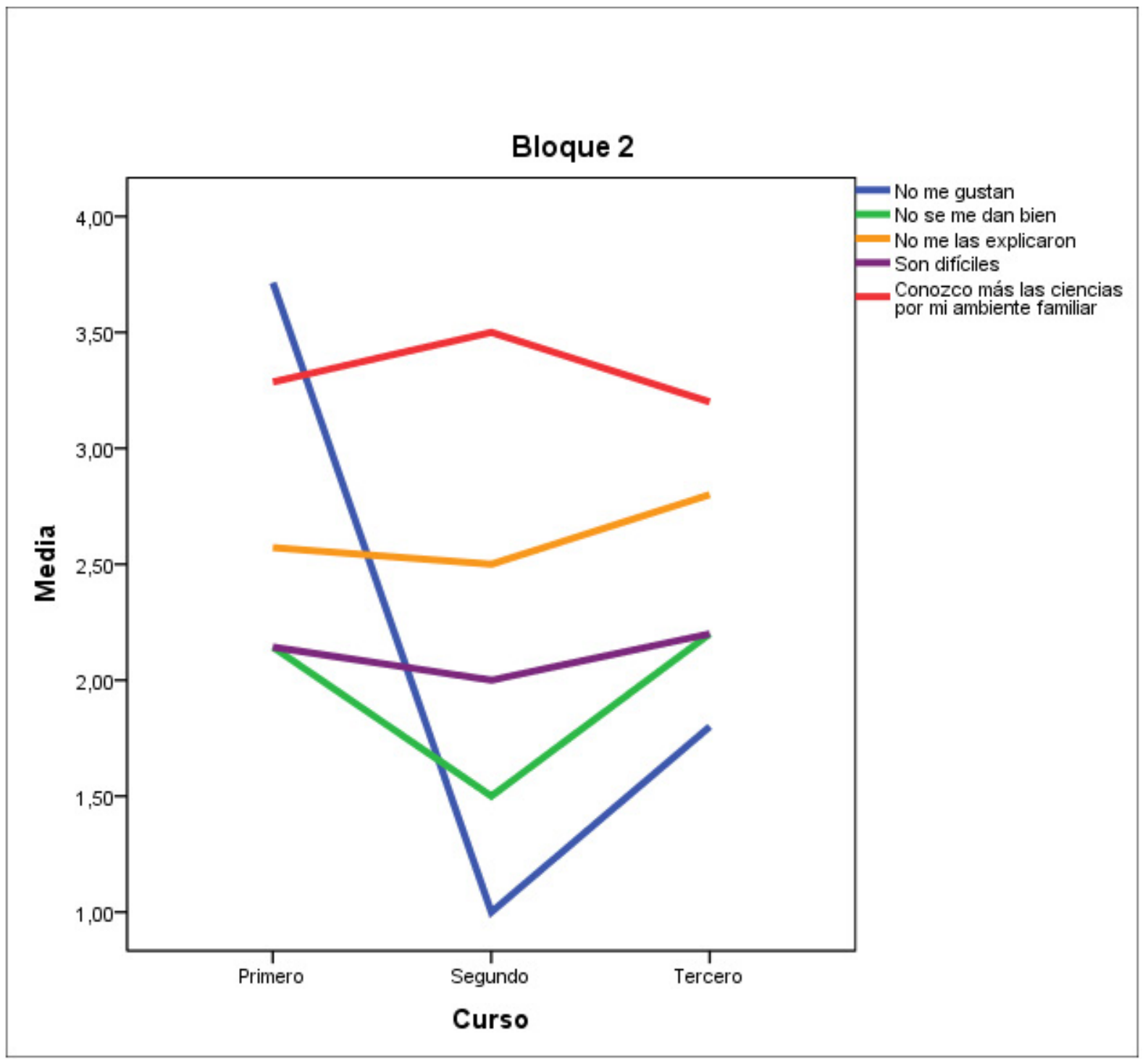

